\title{
Specimen Preparation Considerations for 2D Dopant Profile Determination in Semiconductor Devices by Electron Holography
}

\author{
Kil-Soo Ko, Ji-Ping Zhou, and Lew. Rabenberg
}

Texas Materials Institute, The University of Texas at Austin, Austin, TX 78712

The two-dimensional (2D) dopant profile within a transistor is of great interest to the semiconductor industry.[1] As the downsizing of semiconductor devices continues, a reliable technique to determine the distribution of dopants within submicron devices becomes increasingly more desirable. Electron holography is one technique that can determine dopant distributions; it can be very helpful for confirmation of device design and simulation.[2]

The best specimen preparation method for off-axis electron holography in TEM is not yet established. This poster discusses the use of a focused ion beam (FIB) tool to cut specimens and support them on a $\mathrm{Cu}$ mesh with $30 \mathrm{~nm}$ carbon support film. In this method, the carbon support eliminates the need for carbon coating of the specimen to avoid charging.

For this work, off-axis electron holography is used to investigate the electrostatic potential in semiconductor devices with known dopant profiles. A p-type MOSFET from a dynamic random access memory on $\mathrm{Si}(100)$ wafer is analyzed by electron holography. The specimens are carefully thinned by single beam FIB system with a lift-out pick-up system to avoid $\mathrm{Ga}^{+}$ ion damage to specimen. A $200 \mathrm{KV}$ JEOL 2010F TEM equipped with a biprism is used to acquire the electron hologram.

Figure 1 compares the specimen preparation methods that are used for holography. It is difficult to obtain a clean hologram from the $\mathrm{Ar}^{+}$ion milled specimen because of the overlapping hologram from the other side of specimen. A relatively thick glue line is necessary in order to avoid overlapping of the interference waves, but excessively thick glue lines lead to other difficulties.

Figure 2 shows an actual device hologram and the corresponding phase image from a FIBworked specimen on a carbon coated $\mathrm{Cu}$ mesh. The phase image reveals lateral thickness variations across the gate region due to "curtaining" during FIB thinning. The doped well region is also seen in the phase image. Source and drain regions were not well defined due to improper annealing temperature after implantation. The FIB-worked specimen on the 30 
$\mathrm{nm}$ carbon film was charging under the electron beam; this is evident as black contrast on the left side of the phase image.

(a)

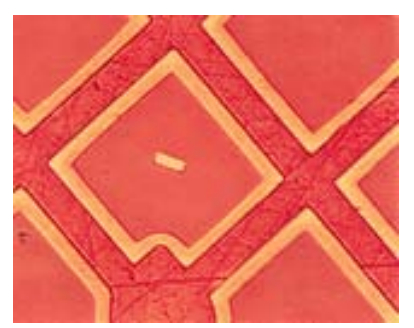

(b)

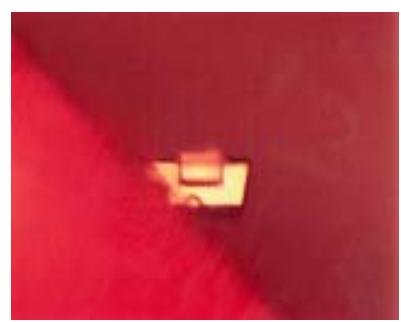

(c)

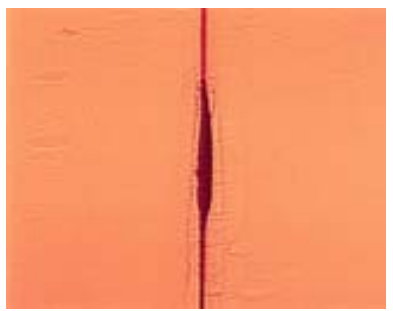

Fig. 1. Optical micrographs showing three different specimen preparation methods. (a). FIB worked specimen on $\mathrm{C}$ coated $\mathrm{Cu}$ mesh, (b). FIB worked specimen on half-cut $\mathrm{Cu}$ grid, (c). Conventionally thinned and dimpled cross sectional specimen finish-milled with $\mathrm{Ar}^{+}$ion milling.

(a)

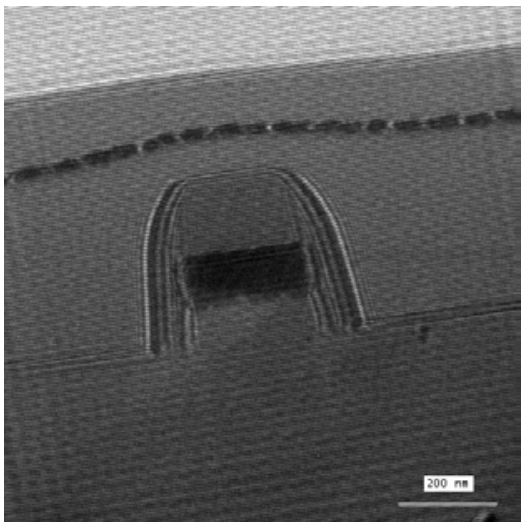

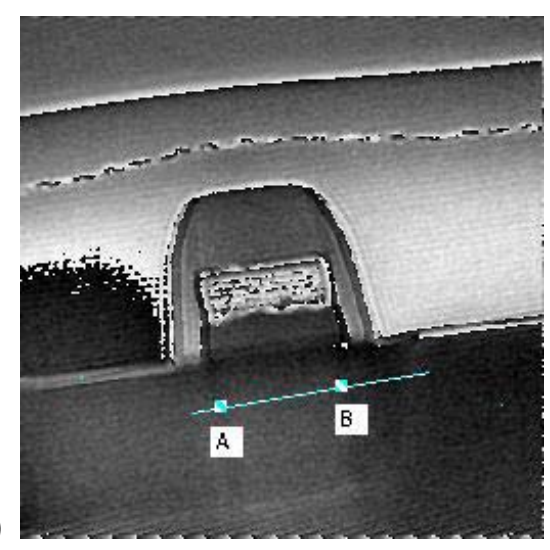

(b)

Fig. 2. Electron hologram (a) and phase image (b) from device.

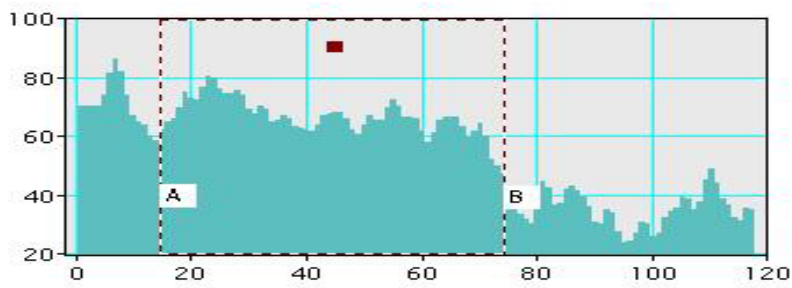

Fig. 3. Profile of phase image along gate line shows thickness variation due to curtaining effect of FIB.

\section{References}

[1] A.C. Diebold et al., J. Vac. Sci. Technol. B 14 (1996) 196.

[2] R. Subrahmanyan, J. Vac. Sci. Technol. B 10 (1992) 358.

[3] Special thanks to Dr M. Kawasaki from JEOL, USA for assistance with setting up the instrument for electron holography. 